\title{
Constraints on MeV dark matter using neutrino detectors and their implication for the $21-\mathrm{cm}$ results
}

\author{
Niki Klop ${ }^{1, *}$ and Shin'ichiro Ando ${ }^{1,2}$ \\ ${ }^{1}$ GRAPPA Institute, University of Amsterdam, 1098 XH Amsterdam, Netherlands \\ ${ }^{2}$ Kavli Institute for the Physics and Mathematics of the Universe (Kavli IPMU, WPI), \\ Todai Institutes for Advanced Study, University of Tokyo, Kashiwa, Chiba 277-8583, Japan
}

(Received 5 September 2018; published 5 November 2018)

\begin{abstract}
The recent results of the EDGES Collaboration indicate that during the era of reionization, the primordial gas was much colder than expected. The cooling of the gas could be explained by interactions between dark matter (DM) and particles in the primordial gas. Constraints from cosmology and particle experiments indicate that this DM should be light $(\sim 10-80 \mathrm{MeV})$, carry a small charge $\left(\epsilon \sim 10^{-6}-10^{-4}\right)$, and only make up a small fraction of the total amount of DM. Several constraints on the DM parameter space have already been made. We explore the yet unconstrained region in the case that the millicharged DM makes up 2\% of the total dark matter, through the scenario in which this DM annihilates only into mu and tau neutrinos. We set upper limits on the annihilation cross section using the Super-Kamiokande data, and predict the limits that could be obtained through Hyper-Kamiokande, JUNO and DUNE. We find that data from SuperKamiokande is not yet able to constrain this model, but future experiments might be. We furthermore explore DM annihilation into solely neutrinos in general, giving an update of the current limits, and predict the limits that could be placed with future experiments.
\end{abstract}

DOI: 10.1103/PhysRevD.98.103004

\section{INTRODUCTION}

Early stars are expected to have imprinted their evidence in the cosmic microwave background (CMB). Their ultraviolet light hit the primordial hydrogen gas, resulting in emission at the $21 \mathrm{~cm}$ line. As a response to this, the absorption from $\mathrm{CMB}$ photons by the primordial gas caused a spectral signal in the CMB that we should be able to observe today. The recent $21-\mathrm{cm}$ results of the EDGES Collaboration [1] show an absorption profile that is consistent with the expected effect induced by the early stars, although showing an amplitude twice as large as predicted. This result implies that the temperature of the primordial gas was much lower than expected, or that the temperature of the background radiation was higher than expected [2-4].

A possible explanation consistent with the observed results is the cooling of the gas due to interactions with dark matter (DM) [1,5-8], which is causing a lot of excitement in the field. The possibility of such a DM interaction was studied in Refs. [9-11], in which multiple constraints are

*1.b.klop@uva.nl

Published by the American Physical Society under the terms of the Creative Commons Attribution 4.0 International license. Further distribution of this work must maintain attribution to the author(s) and the published article's title, journal citation, and DOI. Funded by SCOAP ${ }^{3}$. put on the nature of the responsible DM. Using data from a variety of experiments, it was found that most of the parameter space that is consistent with the $21-\mathrm{cm}$ observations is ruled out [10] (see also Ref. [12]). The DM responsible for the cooling could only make up a small fraction of the total DM, $\sim 0.3-2 \%$, and their mass lies in the range of $\sim 10-80 \mathrm{MeV}$. Furthermore, the DM should carry a small electric charge on the order of $\epsilon \sim 10^{-6}-10^{-4}$.

However, assuming that DM interacts with baryons mediated by only photons produces too much DM through the thermal freeze-out mechanism. In order to circumvent this issue, DM must have at least one more interaction channel with the standard model particles. The simplest possibility that has not been ruled out yet is that the DM interacts with lepton number $L_{\mu}-L_{\tau}$ via either a scalar or vector mediator [10]. This model is hard to constrain with lab experiments because DM does not interact with electrons, and especially for DM lighter than muons (as it is of main interest here), DM can annihilate only into muon and tau neutrinos.

We investigate this scenario, exploring the yet unconstrained parameter space in the energy range of $\sim 10-100 \mathrm{MeV}$. Through flavor mixing, $\nu_{e}$ and $\bar{\nu}_{e}$ have been generated when the neutrinos reach the Earth, which makes it possible for detectors such as Super-Kamiokande (SK) [13] to detect them through charged-current interactions. These neutrinos will show a very specific spectral feature; e.g., in the simplest model investigated in Ref. [10], 
DM annihilation will produce a neutrino line at its mass $(\chi \chi \rightarrow \nu \bar{\nu})$. The energy range of $\sim 10-100 \mathrm{MeV}$, where there are solar, reactor, and atmospheric neutrino backgrounds as well as cosmic-ray muons, has been studied well especially for detecting the diffuse supernova neutrino background [14-16].

We obtain upper limits on the annihilation cross section of this DM in the case that it makes up 2\% of the total DM, the model that could explain the EDGES result, using several years of SK data. We also predict the upper limits that could be obtained by the future experiments HyperKamiokande (HK) [17], the Deep Underground Neutrino Experiment (DUNE) [18] and the Jiangmen Underground Neutrino Observatory (JUNO) [19]. A DM model like this, in which DM only annihilates into neutrinos, but makes up the entire amount of DM, has been studied before [20,21], obtaining upper limits on the cross section using the SK data. We also obtain updated upper limits for this scenario.

The paper is organized as follows. In Sec. II, we discuss millicharged DM and the DM scenario we investigate. In Sec. III, we determine the neutrino flux coming from the annihilation of this millicharged DM, while in Sec. IV, we explain the analysis we perform. In Secs. V and VI, we discuss our results and conclusions respectively.

\section{THEORY}

\section{A. Millicharged DM}

There are some requirements for the properties of DM that need to hold in order for it to be responsible for the extensive cooling of the primordial hydrogen gas [10]. Due to the equipartition theorem, the DM particles should be relatively light. Furthermore, models in which the cross section for dark matter scatterings with gas is independent of the velocity can already be ruled out by constraints from observations of the CMB.

To fulfil these requirements, the mediator of the dark matter-baryon interactions should be lighter than the temperature of the gas at $z \sim 17$. New light mediators in the mass range required to explain the EDGES result are ruled out [22,23], and their contribution to the radiation part of the energy density would exceed the current constraints [24]. When the DM carries a small electric charge, it could couple to the photon. Reference [9] found that, in order to cool the gas sufficiently, the following condition for the electric charge should be fulfilled:

$$
\epsilon \approx 1.7 \times 10^{-4}\left(\frac{m_{\chi}}{300 \mathrm{MeV}}\right)\left(\frac{10^{-2}}{f_{\chi}}\right)^{3 / 4},
$$

where $\epsilon \equiv e_{\chi} / e$ is the electric charge of the millicharged DM particle, $m_{\chi}$ is its mass and $f_{\chi}$ is its mass fraction of the total DM. The existence of millicharged DM is already constrained by multiple experiments and astrophysical data, leaving only a small open window in its possible parameter space, with a mass $m_{\chi}$ of $\sim 10-80 \mathrm{MeV}$, and a total DM fraction of $f_{\chi} \sim 0.003-0.02$ [10].

However, the annihilation of millicharged DM particles through the exchange of a photon is not sufficient to yield the desired energy density for the particle, $f_{\mathrm{DM}} \Omega_{\mathrm{CDM}}$. Therefore, some additional annihilation needs to take place through a new mediator. Here we specifically study the case of a vector mediator $V$, while the results for the scalar mediator are essentially the same. Reference [10] found that annihilation through the new vector mediator $V$ into standard model fermions is excluded if it couples to all flavors. This leads to the consideration of annihilation into mainly neutrinos. When the new vector $V$ is related to the gauge group $U(1)_{L_{\mu}-L_{\tau}}$, only coupling to muons, taus, muon neutrinos and tau neutrinos is possible. Since $V$ does not couple to electrons, there are currently few constraints from experiments. The annihilation cross section to any neutrino flavor for such a model is given by

$$
\langle\sigma v\rangle=\frac{g_{\nu}^{2} g_{\chi}^{2} m_{\chi}^{2} \kappa}{2 \pi\left(4 m_{\chi}^{2}-m_{V}^{2}\right)^{2}},
$$

where $g_{\nu}$ and $g_{\chi}$ are the gauge coupling constants of the neutrino and DM particle, respectively, and $\kappa=1\left(v^{2} / 6\right)$ for fermion (scalar) DM. We put constraints on this model by evaluating the SK data, and make predictions for some future experiments.

Besides this, we also consider DM annihilation into neutrinos in a broader sense. If dark matter only annihilates into neutrinos, this would be harder to detect than the cases where gamma rays are produced. The limits on the annihilation cross section in this case will therefore be the most conservative ones, and therefore interesting to investigate. We update the limits obtained in Ref. [20], calculating the limits in both the case that DM annihilates to all three neutrino flavors, and the case where it only annihilates into muon and tau neutrinos as discussed above.

\section{NEUTRINO FLUX FROM DARK MATTER ANNIHILATION}

The final flavor ratio at Earth for pure $\nu_{\mu}$ and $\nu_{\tau}$ channels is $1: 2: 2$. When Galactic DM annihilates into $\nu_{\mu}$ and $\nu_{\tau}$, the expected monochromatic flux of electron (anti)neutrinos at Earth will therefore be given by

$$
\frac{\mathrm{d} \phi}{\mathrm{d} E_{\nu}}=\frac{\langle\sigma v\rangle}{2} \mathcal{J}_{\mathrm{avg}} \frac{R_{\mathrm{sc}} \rho_{0}^{2} f_{\chi}^{2}}{m_{\chi}^{2}} \frac{1}{5} \delta\left(E_{\nu}-m_{\chi}\right),
$$

in the case of Majorana DM, where $\sigma$ is the annihilation cross section, $m_{\chi}$ is the mass of the DM particle, $E_{\nu}$ is the neutrino energy, $\mathcal{J}_{\text {avg }}$ is the angular-averaged " $J$-factor" of the Milky Way, for which we use the canonical value $\mathcal{J}_{\text {avg }}=5[25], R_{\mathrm{sc}}=8.5 \mathrm{kpc}$ is the scale radius of the Milky Way, and $\rho_{0}=0.3 \mathrm{GeV} \mathrm{cm}^{-3}$ is the DM density at 


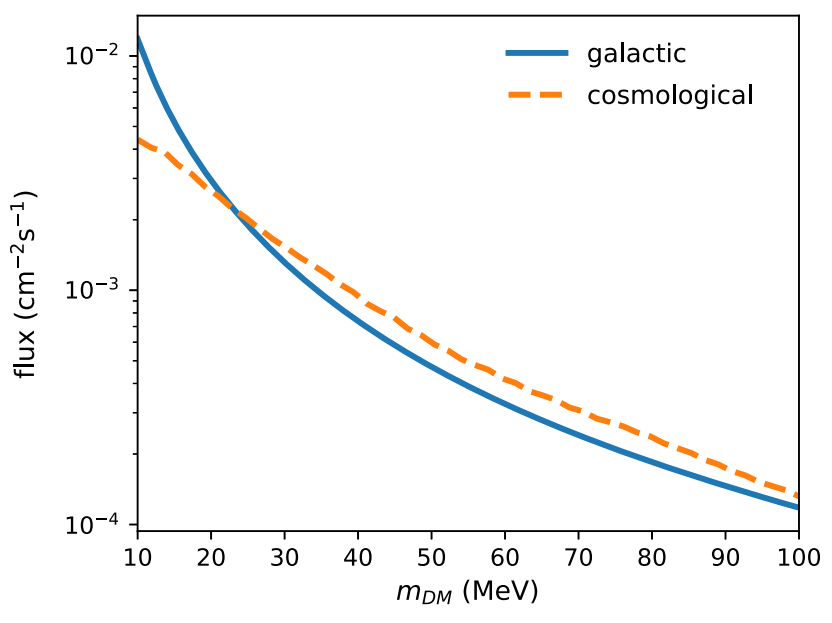

FIG. 1. The total integrated electron neutrino fluxes at Earth of both the cosmological and the Galactic contributions as a function of the DM mass, in the case of $2 \%$ Dirac DM annihilating into only muon and tau neutrinos.

the scale radius. To retrieve the electron (anti)neutrino flux for Dirac DM, Eq. (3) has to be divided by 2.

In the thermal freeze-out scenario, the annihilation cross section at freeze-out required to leave the correct relic abundance of $\mathrm{MeV} \mathrm{DM}$ is given by

$$
\langle\sigma v\rangle=\frac{5 \times 10^{-27} \mathrm{~cm}^{3} \mathrm{~s}^{-1}}{\Omega_{\chi} h^{2}},
$$

for Majorana fermion DM, and is twice as large for Dirac fermion DM for masses below $\mathrm{GeV}$ [26]. Since $\Omega_{\chi} h^{2} \approx 0.1 f_{\chi}$, the targeted annihilation cross section is $\langle\sigma v\rangle \approx 2.5 \times$ $10^{-24}\left(f_{\chi} / 0.02\right)^{-1} \mathrm{~cm}^{3} \mathrm{~s}^{-1}$ and $5 \times 10^{-24}\left(f_{\chi} / 0.02\right)^{-1} \mathrm{~cm}^{3} \mathrm{~s}^{-1}$ for Majorana and Dirac DM respectively.

Besides the Galactic neutrino flux, we also take into account the contribution to the flux coming from DM annihilations outside our galaxy. We adopt the calculation of Ref. [27] with the most recent model of substructure boost [28]. This cosmological neutrino flux is of the same order of magnitude as the Galactic contribution, but it is nonmonochromatic due to its redshift. In Fig. 1, the total integrated fluxes of both the cosmological and the Galactic contributions are shown as functions of the DM mass.

\section{THE ANALYSIS}

We set upper limits on the DM annihilation cross section using the latest SK data [13], and predict the upper limits that could be obtained by the future experiments HyperKamiokande [17], DUNE [18] and JUNO [19]. We use the SK data from the first three data periods [13], which contain 2853 days of data taking in total, in the energy range of $16-88 \mathrm{MeV}$, considering 18 bins with a width of $4 \mathrm{MeV}$. The expected number of events at the detector coming from DM annihilation is calculated through

$$
N_{\text {events }}=\sigma_{\text {det }} \phi N_{\text {target }} \epsilon_{\text {det }} t
$$

where $\sigma_{\text {det }}$ is the detection cross section, $\phi$ is the neutrino flux, $N_{\text {target }}$ is the number of target particles in the detector, $\epsilon_{\text {det }}$ is the efficiency of the detector which we get from Ref. [13], and $t$ is the exposure time. SK is a $22.5 \mathrm{kton}$ water Cherenkov detector [13], detecting neutrinos through the measurement of Cherenkov radiation from relativistic electrons and positrons. The relevant detection channels in our energy range are inverse beta decay $\left(\bar{\nu}_{e}+p \rightarrow e^{+}+n\right)$, and the absorption of $\nu_{e}$ and $\bar{\nu}_{e}$ by oxygen in charged-current interactions $\left(\nu_{e}^{(-)}+{ }^{16} \mathrm{O} \rightarrow e^{ \pm}+\mathrm{X}\right)$. The energies of electrons and positrons produced by these interactions are $E_{e}=E_{\nu}-1.3 \mathrm{MeV}\left(\bar{\nu}_{e} p\right), E_{\nu}-15.4 \mathrm{MeV}\left(\nu_{e} O\right)$, and $E_{\nu}-11.4 \mathrm{MeV}\left(\bar{\nu}_{e} O\right)$. The cross sections for these detection channels are taken from Refs. [29,30]. To correct for the energy resolution of the experiment, we smear the expected electron (positron) spectrum with a Gaussian function, using an energy resolution of width

$$
\sigma=0.40 \mathrm{MeV} \sqrt{E / \mathrm{MeV}}+0.03 E,
$$

that we take from Ref. [20]. We perform a $\chi^{2}$ analysis of the expected number of events compared to the data, and calculate the upper limit at the $90 \%$ confidence level. We consider four different backgrounds coming from atmospheric neutrinos, which we also take from Ref. [13]. This background data is taken from the first running phase of SK, SK-I. We rescale it to the entire exposure time that we consider of 2853 days.

The future experiments that we consider show a lot of improvement in several ways. The invisible muon background originating from $\nu_{\mu} / \bar{\nu}_{\mu}$ charged-current events, which is the biggest background in SK below $\sim 40 \mathrm{MeV}$, might be significantly decreased in measurements of future water Cherenkov detectors by adding gadolinium [31]. In our analysis for Hyper-Kamiokande, we assume a reduction of this background by $80 \%$. In the case of JUNO, this background is removed in total through the implementation of an extra system for cosmic muon detection and background reduction [32]. In the case of DUNE, this background is removed (e.g., see Ref. [33]). For our prediction, we consider an exposure time of 3000 days for each detector. We use the same background data as in SK, rescaling it to the right exposure time and the size of the specific experiment. In the case of Hyper-Kamiokande, a 520-kton upgrade of SK [17] with a fiducial volume of $374 \mathrm{kton}$, the same energy resolution is used [Eq. (6)].

DUNE is a 46.4-kton liquid argon detector [34]. The relevant detection channels are the detection of electron (anti)neutrinos through charged-current interactions $\left(\nu_{e} / \bar{\nu}_{e}+{ }^{40} \mathrm{Ar} \rightarrow e^{-} / e^{+}+\mathrm{A}^{\prime}+n N\right) \quad[30]$, where $n N$ are the emitted nuclei and $\mathrm{A}^{\prime}$ is the remaining nucleus. JUNO is a 20-kton lab-based liquid scintillator [19]. The detection channels relevant for JUNO are again inverse beta decay 
$\left(\bar{\nu}_{e}+p \rightarrow e^{+}+n\right)$, and the capture of electron (anti) neutrinos on ${ }^{12} \mathrm{C}$ in charged-current interactions $\left(\bar{\nu}_{e}+{ }^{12} \mathrm{C} \rightarrow{ }^{12} \mathrm{~B}+e^{+} / \nu_{e}+{ }^{12} \mathrm{C} \rightarrow{ }^{12} \mathrm{~N}+e^{-}\right) \quad$ [30]. The energy resolutions for both JUNO and DUNE are significantly better than the water Cherenkov detectors. For DUNE, the energy resolution is given by [35]

$$
\sigma=0.025 \mathrm{MeV} \sqrt{E / \mathrm{MeV}}+0.060 E
$$

For JUNO, the energy resolution we use is given by [36]

$$
\sigma=0.03 \mathrm{MeV} \sqrt{E / \mathrm{MeV}} .
$$

To predict the upper limit at the $90 \%$ confidence level, we use the Python tool swordfish $[37,38]$ that can, among other things, predict upper limits based on Poisson statistics, resulting in approximately the mean value of the results that one would get by performing a Monte Carlo simulation. Besides this, in the case of Hyper-Kamiokande, we explicitly perform a Monte Carlo simulation to obtain the full scope of possible values. We furthermore consider the reach of a hypothetical experiment with the size of Hyper-Kamiokande and the specifications of JUNO, which would result in a very strong experiment.

We obtain the upper limits for several cases. Besides the case of a millicharged Dirac DM particle responsible for $2 \%$ of the total DM, annihilating into only muon and tau neutrinos, we also consider the situation where $100 \%$ of DM only annihilates into neutrinos. In this situation we consider two subcases. In the first case, the DM has the same properties as in the $2 \%$ situation. In the second case, we consider Majorana DM, annihilating into all three neutrino flavors. Since in this case the expected flavor ratio at Earth is $1: 1: 1$, the expected neutrino flux is given by

$$
\frac{\mathrm{d} \phi}{\mathrm{d} E_{\nu}}=\frac{\langle\sigma v\rangle}{2} \mathcal{J}_{\text {avg }} \frac{R_{\mathrm{sc}} \rho_{0}^{2}}{m_{\chi}^{2}} \frac{1}{3} \delta\left(E_{\nu}-m_{\chi}\right) .
$$

The latter case is similar to ordinary weakly interacting massive particle (WIMP) DM, except that the neutrino-only restriction makes it harder to detect. Therefore, the upper limits obtained in this situation will be the most conservative constraints for MeV WIMP DM annihilation.

\section{RESULTS}

In the top panel of Fig. 2, the upper limits are plotted for the case of the $2 \%$ millicharged DM. The black dashed line is the cross section corresponding to the DM model that could explain the EDGES results. The SK limit is not strong enough to rule out the millicharged DM model. Based on 3000 running days, the predictions show that Hyper-Kamiokande, JUNO and DUNE cannot reach the desired limit as well. However, the actual data will probably induce some fluctuations, possibly resulting in a stronger limit, as can be seen from the behavior of the Monte Carlo region of Hyper-Kamiokande, compared to its predicted line. The strongest limit comes from DUNE. Running the detector long enough might result in strong enough limits to constrain the millicharged DM model. A combined analysis of the data of these experiments could result in a stronger limit by up to a factor of 2 . In the bottom panel of Fig. 2, we show the limits in terms of the coupling constants and the masses of the dark matter particle and the new mediator, $\left(g_{\chi} g_{f}\right)^{2}\left(m_{\chi} m_{V}\right)^{4}$, specific to our DM model.

Figure 3 shows the SK limits both with and without taking the extragalactic DM annihilation flux into account. We note that the Galactic flux has a substantial contribution to the limit.

We note that the most recent CMB (re)analysis found that the fraction of millicharged DM might be constrained even more tightly, $f_{\chi} \lesssim 0.4 \%$ [39] (and references therein). In the case of $f_{\chi}=0.4 \%$, our limits get weaker by a factor of $(2 / 0.4)^{2}=25$ [Eq. (3)]. However, the annihilation cross
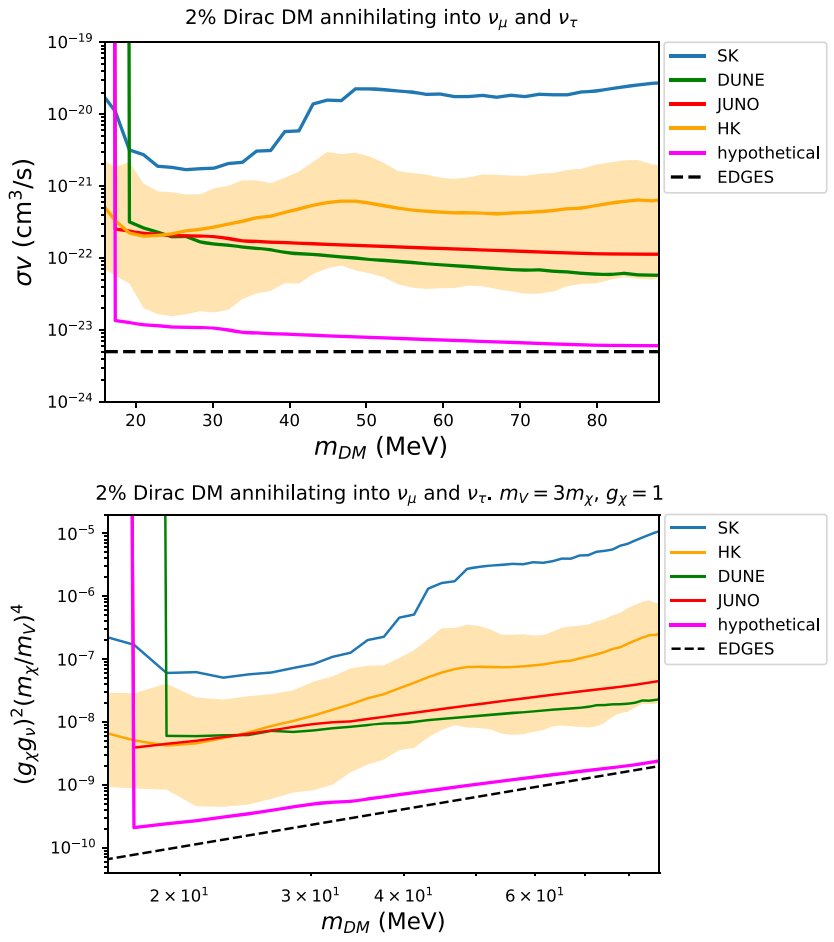

FIG. 2. Upper limits on the annihilation cross section $\langle\sigma v\rangle$ of millicharged Dirac DM into only muon and tau neutrinos, making up $2 \%$ of the total DM, as a function of the DM mass (upper panel). The lower panel shows the annihilation cross section in terms of the coupling constants, the DM mass, and the mass of the new mediator $V$. The black dashed line is the cross section induced by the EDGES results, in the case of Dirac DM. The blue line comes from the analysis of 2853 days of SK data. The other lines are predictions using swordfish $[37,38]$. The orange band shows the region between the minimum and maximum upper limit values predicted for Hyper-Kamiokande through a Monte Carlo simulation. 


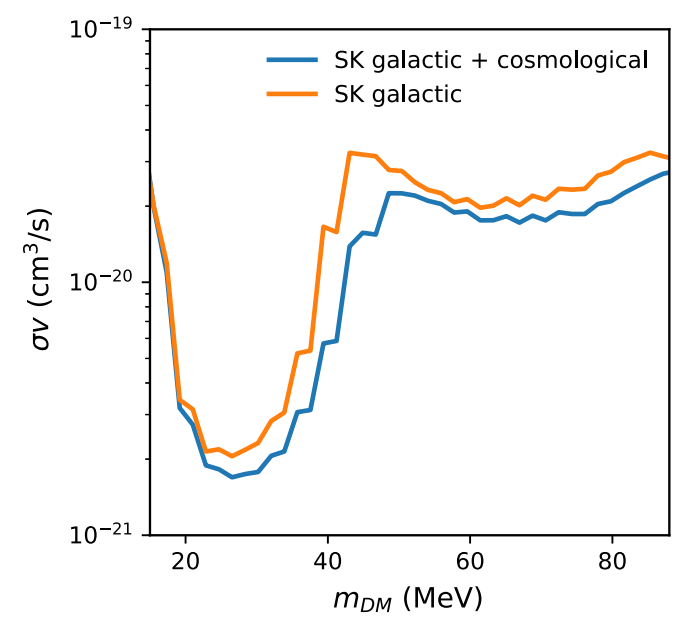

FIG. 3. Upper limits on the annihilation cross section of millicharged Dirac DM, making up $2 \%$ of the total DM, as a function of the DM mass, computed from the analysis of 2853 days of SK data. The orange line only contains the Galactic contribution to the neutrino flux coming from DM annihilations, while the blue line also includes the extragalactic contribution.
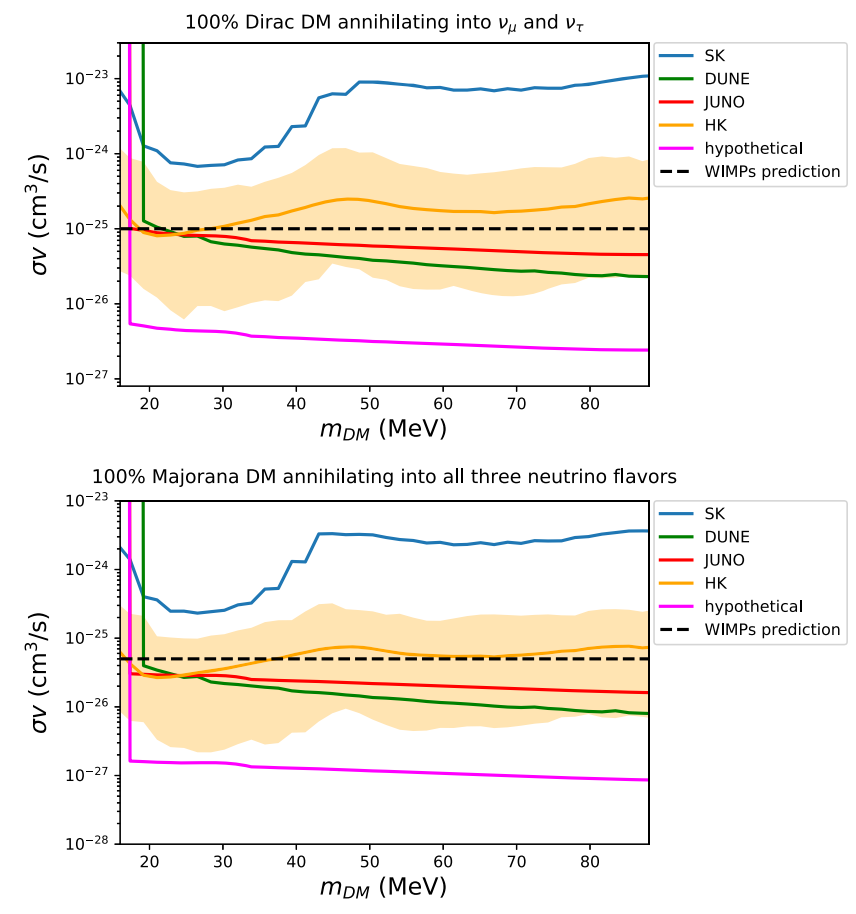

FIG. 4. Upper limits on the annihilation cross section of millicharged Majorana DM, making up $100 \%$ of DM, as a function of the DM mass. The upper panel shows the case of Dirac DM annihilating into only muon and tau neutrinos. The lower panel shows the case of Majorana DM annihilating into all three neutrino flavors with equal fraction. The black dashed line is the preferred cross section for WIMPs, in the case of Dirac (Majorana) DM in the upper (lower) panel. The blue line comes from the analysis of 2853 days of SK data. The other lines are predictions using swordfish $[37,38]$. The orange band shows the region between the minimum and maximum upper limit values for HyperKamiokande predicted through a Monte Carlo simulation. section required to explain the relic abundance becomes larger by a factor of $2 / 0.4=5$ [see Eq. (4) and subsequent sentences]. Hence our limits on the annihilation cross section relative to its canonical value will be weakened by a factor of 5 .

Finally, besides exploring the millicharged DM model, we explore two more generic cases, where $100 \%$ of DM annihilates into neutrinos. First, we study the same Dirac DM annihilating into only muon and tau neutrinos, whose results are shown in the top panel of Fig. 4. The second case is Majorana DM annihilating into three neutrino flavors with equal fraction, shown in the bottom panel of Fig. 4. Since neutrinos are harder to detect than gamma rays, this results in a more conservative estimate, and hence the most general constraints on the DM annihilation [25]. While the current limit of SK could not yet constrain the WIMP prediction, in both of these cases, Hyper-Kamiokande, JUNO and DUNE will certainly be able to do so. We note that our updated limit is weaker than the limit found in Ref. [20] based on the previous data set of SK [40] by a factor of a few.

\section{CONCLUSIONS}

The recent results of EDGES suggest that the primordial gas underwent extensive cooling from some additional DM species. Several constraints on the DM parameter space have already been made. We explored the yet unconstrained region in the case that millicharged DM makes up 2\% of the total dark matter, through the scenario in which this DM interacts with the standard model through the $\mu-\tau$ lepton number. This additional interaction is motivated by the thermal freeze-out scenario to explain the correct relic density, and also by the fact that it is largely unconstrained. If this DM has a mass of $10-100 \mathrm{MeV}$ as suggested by the EDGES measurement, it annihilates only into mu and tau neutrinos.

By calculating the neutrino flux from the Galactic and extragalactic halos and comparing with existing data, we found that data from Super-Kamiokande are not yet able to constrain this model. We however found that future experiments might be able to detect neutrinos from this particular DM species. The hypothetical experiment that we studied with a size comparable to Hyper-Kamiokande and energy resolution comparable to JUNO or DUNE would be able to reach the desired limits. Although such an experiment is not scheduled to be built in the near future, there has been a Europe-wide initiative to study the possibility of an experiment with a size of the right order of magnitude [41]. We furthermore provided updated limits on the annihilation cross section for a more general WIMP DM model in the mass range 16-88 $\mathrm{MeV}$, using (expected) data from the current and future neutrino experiments. We found that the current data of SK cannot yet put constraints on the WIMPs prediction, but the future experiments HyperKamiokande, DUNE and JUNO will be capable of this. 


\section{ACKNOWLEDGMENTS}

We thank Thomas Edwards and Mauricio Bustamante for the useful discussions. This work was supported by the Foundation for Fundamental Research on Matter (FOM) through the FOM Program (N. K. and S. A.), and partly financed by Japan Society for the Promotion of Science (JSPS) KAKENHI Grants No. JP17H04836, No. JP18H04340, and No. JP18H04578 (S. A.).

[1] J. D. Bowman, A. E. E. Rogers, R. A. Monsalve, T. J. Mozdzen, and N. Mahesh, Nature (London) 555, 67 (2018).

[2] M. Pospelov, J. Pradler, J. T. Ruderman, and A. Urbano, Phys. Rev. Lett. 121, 031103 (2018).

[3] S. Fraser et al., Phys. Lett. B 785, 159 (2018).

[4] C. Feng and G. Holder, Astrophys. J. 858, L17 (2018).

[5] R. Barkana, Nature (London) 555, 71 (2018).

[6] A. Fialkov, R. Barkana, and A. Cohen, Phys. Rev. Lett. 121, 011101 (2018).

[7] H. Tashiro, K. Kadota, and J. Silk, Phys. Rev. D 90, 083522 (2014).

[8] J. B. Muoz, E. D. Kovetz, and Y. Ali-Hamoud, Phys. Rev. D 92, 083528 (2015).

[9] J. B. Muoz and A. Loeb, Nature (London) 557, 684 (2018).

[10] A. Berlin, D. Hooper, G. Krnjaic, and S. D. McDermott, Phys. Rev. Lett. 121, 011102 (2018).

[11] E. D. Kovetz et al., arXiv:1807.11482.

[12] G. Magill, R. Plestid, M. Pospelov, and Y. D. Tsai, arXiv: 1806.03310 .

[13] K. Bays et al. (Super-Kamiokande Collaboration), Phys. Rev. D 85, 052007 (2012).

[14] S. Ando and K. Sato, New J. Phys. 6, 170 (2004).

[15] J. F. Beacom, Annu. Rev. Nucl. Part. Sci. 60, 439 (2010).

[16] C. Lunardini, Astropart. Phys. 79, 49 (2016).

[17] K. Abe et al. (Hyper-Kamiokande Collaboration), arXiv: 1805.04163.

[18] J. Strait et al. (DUNE Collaboration), Proceedings, 17th International Workshop on Neutrino Factories and Future Neutrino Facilities (NuFact15) : Rio de Janeiro, Brazil, 2015 (2015).

[19] V. Antonelli and L. Miramonti (JUNO Collaboration), Proc. Sci., NEUTEL2017 (2018) 056.

[20] S. Palomares-Ruiz and S. Pascoli, Phys. Rev. D 77, 025025 (2008).

[21] A. Olivares-Del Campo, S. Palomares-Ruiz, and S. Pascoli, Implications of a dark matter-neutrino coupling at HyperKamiokande, in Proceedings of the 53rd Rencontres de Moriond (EW 2018), La Thuile, 2018, [arXiv:1805.09830].
[22] E. G. Adelberger, B R. Heckel, S. Hoedl, C. D. Hoyle, D. J. Kapner, and A. Upadhye, Phys. Rev. Lett. 98, 131104 (2007).

[23] D. J. Kapner, T. S. Cook, E. G. Adelberger, J. H. Gundlach, B. R. Heckel, C. D. Hoyle, and H. E. Swanson, Phys. Rev. Lett. 98, 021101 (2007).

[24] P. A. R. Ade et al. (Planck Collaboration), Astron. Astrophys. 594, A13 (2016).

[25] H. Yuksel, S. Horiuchi, J. F. Beacom, and S. Ando, Phys. Rev. D 76, 123506 (2007).

[26] G. Steigman, B. Dasgupta, and J. F. Beacom, Phys. Rev. D 86, 023506 (2012).

[27] S. Ando and E. Komatsu, Phys. Rev. D 87, 123539 (2013).

[28] N. Hiroshima, S. Ando, and T. Ishiyama, Phys. Rev. D 97, 123002 (2018).

[29] A. Strumia and F. Vissani, Phys. Lett. B 564, 42 (2003).

[30] S. Skadhauge and R. Zukanovich Funchal, J. Cosmol. Astropart. Phys. 04 (2007) 014.

[31] J. F. Beacom and M. R. Vagins, Phys. Rev. Lett. 93, 171101 (2004).

[32] H. Lu and E. Baussan (JUNO Collaboration), J. Phys. Conf. Ser. 888, 012088 (2017).

[33] A. G. Cocco, A. Ereditato, G. Fiorillo, G. Mangano, and V. Pettorino, J. Cosmol. Astropart. Phys. 12 (2004) 002.

[34] V. A. Kudryavtsev et al. (DUNE Collaboration), J. Phys. Conf. Ser. 718, 062032 (2016).

[35] V. De Romeri, E. Fernandez-Martinez, and M. Sorel, J. High Energy Phys. 09 (2016) 030.

[36] F. An et al. (JUNO Collaboration), J. Phys. G 43, 030401 (2016).

[37] T. D. P. Edwards and C. Weniger, arXiv:1712.05401.

[38] T. D. P. Edwards and C. Weniger, J. Cosmol. Astropart. Phys. 02 (2018) 021.

[39] K. K. Boddy et al., arXiv:1808.00001.

[40] M. Malek et al. (Super-Kamiokande Collaboration), Phys. Rev. Lett. 90, 061101 (2003).

[41] D. Angus et al. (LAGUNA Collaboration), in Proceedings of the European Strategy for Future Neutrino Physics Workshop, Geneva, Switzerland, 2009, edited by A. Blondel and F. Dufour (CERN, Geneva, 2010), p. 226. 